\title{
Multifractal dimension of chaotic attractors in a driven semiconductor superlattice
}

\author{
O. M. Bulashenko \\ Departament de Fiśica Fonamental, Universitat de Barcelona, Diagonal 647, E-08028 Barcelona, Spain \\ K. J. Luo, H. T. Grahn,* and K. H. Ploog \\ Paul-Drude-Institut für Festkörperelektronik, Hausvogteiplatz, 5-7, D-10117 Berlin, Germany \\ L. L. Bonilla \\ Escuela Politécnica Superior, Universidad Carlos III de Madrid, Avenida de la Universidad 20, E-28911 Leganés, Spain
}

(Received 19 January 1999; revised manuscript received 16 April 1999)

\begin{abstract}
The multifractal dimension of chaotic attractors has been studied in a weakly coupled superlattice driven by an incommensurate sinusoidal voltage as a function of the driving voltage amplitude. The derived multifractal dimension for the observed bifurcation sequence shows different characteristics for chaotic, quasiperiodic, and frequency-locked attractors. In the chaotic regime, strange attractors are observed. Even in the quasiperiodic regime, attractors with a certain degree of strangeness may exist. From the observed multifractal dimensions, the deterministic nature of the chaotic oscillations is clearly identified. [S0163-1829(99)03032-5]
\end{abstract}

\section{INTRODUCTION}

Dissipative dynamical systems (e.g., current oscillations in a solid-state system driven by an ac voltage) are characterized by the attraction of all trajectories passing through a certain domain of phase space towards a geometrical object called an "attractor.", 1,2 The attractor can be a single point, a number of isolated points, or an extended object in phase space. Therefore, the structure of the attractor is crucial for the understanding of the dynamical behavior of a system. ${ }^{1,2}$ In a physical experiment, one usually employs the so-called first return maps or Poincaré maps to visualize and analyze the underlying attractors. ${ }^{1,2}$ For example, for frequency locking, which corresponds to periodic oscillations, the attractor consists of a set of discrete points so that the dimension of the attractor is zero. For quasiperiodic oscillations, the Poincare map usually consists of a loop with a homogeneous point density so that the dimension of the attractor is one. In the chaotic regime, the structure of the attractor is called "strange." The dimension of the attractor becomes now a fractal instead of an integer number. Strange attractors are usually characterized by a multifractal dimension $D_{q}$, which is a measure of the strangeness of the attractor. ${ }^{3,4}$

Resonant tunneling in weakly coupled semiconductor superlattices (SL's) results in a negative differential velocity (NDV) in the drift velocity vs electric-field characteristics. ${ }^{5-7}$ Due to the presence of the NDV, the vertical transport in the SL's exhibits nonlinear properties, which result in such phenomena as domain formation, ${ }^{8}$ multistability, ${ }^{9}$ and selfsustained oscillations. ${ }^{6,7,10}$ In this nonlinear system, chaos has also been studied theoretically ${ }^{11}$ and experimentally. ${ }^{12,13}$ Recently, an explosive bifurcation to chaos has been experimentally observed in the power spectra of a driven SL and unambiguously identified using Poincare maps. ${ }^{13}$ During the bifurcation sequence from frequency-locking to chaos, the Poincaré map expands from isolated points to connected branches, indicating that the dimension of the underlying attractors increases with increasing amplitude of the driving voltage. When the system enters the chaotic regime, the Poincaré map becomes more complex indicating that the attractor acquires a higher dimension. In order to prove the increase of the attractor dimension and to obtain additional information about the underlying chaos, it is necessary to quantitatively characterize the dimension of the attractors as a function of the external driving voltage amplitude.

In this paper we will report on the multifractal dimension of the attractors for a weakly coupled SL under an external driving voltage. The obtained fractal dimensions unambiguously identify the presence of strange attractors and the deterministic nature of the chaotic oscillations. We also find that strange attractors may exist in the quasiperiodic region.

The paper is organized as follows. In Sec. II the experimental bifurcation route to chaos in a driven SL is briefly reviewed. In Sec. III the dimension of the attractors in the observed bifurcation sequence is derived from the experimental Poincaré maps and discussed. Finally, a short summary is given in Sec. IV.

\section{EXPERIMENTAL FACTS}

In the experiments the dc bias is fixed and the sample is driven by an incommensurate ac voltage. The detailed information about the samples and the performed experiments can be found in Ref. 13. In this paper we will discuss the bifurcation route at the dc bias of $6.574 \mathrm{~V}$, where the current self-oscillations exhibit an intrinsic fundamental frequency $f_{0}$ of $30.5 \mathrm{MHz}$. In the experiment, the driving frequency $\left(f_{d}\right)$ is set to the golden mean $(1+\sqrt{5}) / 2 \approx 1.618$ times $f_{0}$, i.e., $49.4 \mathrm{MHz}$, while the driving amplitude $V_{a c}$ is varied. The observed power spectra as a function of $V_{a c}$ is given in Fig. 1(a) in Ref. 13, which shows the following sequence: quasiperiodicity $\rightarrow$ synchronized chaos $\rightarrow$ frequency locking $\rightarrow$ chaos $\rightarrow$ chaos with higher complexity. The Poincaré maps derived from real-time traces further verify this bifurcation route to chaos. ${ }^{13}$ At small $V_{a c}$, such as 9 and $34 \mathrm{mV}$ shown in Fig. 3 of Ref. 13, the Poincare map consists of a 
closed loop, corresponding to a quasiperiodic attractor. At 39 $\mathrm{mV}$, the loop is split into three extended branches, indicating the appearance of synchronized chaos. By increasing $V_{a c}$ from 39 to $40 \mathrm{mV}$, the system enters a frequency-locked state with a Poincaré map consisting of only three points (they are broadened due to noise). This frequency-locked state exists up to values of $V_{a c}$ of about $50 \mathrm{mV}$, while at 52 $\mathrm{mV}$ two of these three points expand into branches demonstrating the onset of chaos. Within this chaotic regime, a number of chaotic attractors with different complexity are revealed. The Poincaré maps between 50 and $56 \mathrm{mV}$ show that the bifurcation from frequency-locking to chaos is explosive, i.e., it is accompanied by an abrupt increase of the attractor dimension (from three points with zero dimension to an object with finite dimensions).

In Sec. III we will estimate the multifractal dimension of the attractors from the experimental Poincare maps in order to analyze the bifurcation process quantitatively. We will report the results for some typical attractors for at $V_{a c}$ of 9 , $34,40,50,52,56,63$, and $79 \mathrm{mV}$. The Poincaré maps for these driving amplitudes are all shown in Fig. 3 of Ref. 13 except for $50 \mathrm{mV}$. At $50 \mathrm{mV}$, the oscillation is frequency locked, and the Poincare map consists of three points, similar to that for $40 \mathrm{mV}$.

\section{CHARACTERIZATION OF ATTRACTORS}

As mentioned in Sec. I, an attractor can be characterized quantitatively by a multifractal dimension $D_{q}$, where $q$ is a continuous index. If the attractor is a point, line, or twodimensional surface, then $D_{q}$ is 0,1 , or 2 , respectively, independently of the parameter $q$. However, if the attractor becomes more complicated, then its dimension can be a fractal number, whose actual value varies with $q$. In order to determine the fractal dimensions, the attractor is first covered with a grid of cubes. Then the frequency, with which a trajectory visits the various cubes covering the attractor, is calculated in the limit that the trajectory length goes to infinity. ${ }^{3,4}$ This frequency is used to define various fractal dimensions such as the box-counting, information, or correlation dimensions. ${ }^{2}$ As shown previously (cf. Fig. 3 of Ref. 13), the point density varies considerably in different parts of a chaotic attractor, indicating that some regions are much more frequently visited than others. Instead of using a grid of cubes, another equivalent method is to calculate the correlation between different points, a method proposed by Grassberger and Procaccia ${ }^{14}$ for the case of $q=2$ and later generalized for the whole spectrum of dimensions $D_{q} \cdot{ }^{15}$ In this method the multifractal dimension $D_{q}$ is defined as ${ }^{14,15}$

$$
D_{q}=\frac{1}{q-1} \lim _{r \rightarrow 0} \frac{\ln \left[C_{q}(r)\right]}{\ln (r)},
$$

with the generalized correlation function $C_{q}(r)$ given by

$$
C_{q}(r)=\frac{1}{N} \sum_{i=1}^{N}\left[\frac{1}{N} \sum_{j=1}^{N} \theta\left(r-\left|\vec{x}_{i}-\vec{x}_{j}\right|\right)\right]^{q-1},
$$

where $N$ is the number of points, $\vec{x}_{i}$ is a point on the attractor, and $\theta$ is the Heaviside step function. In these equations, $q$ can be any number. $D_{0}$ is known as the capacity dimen-

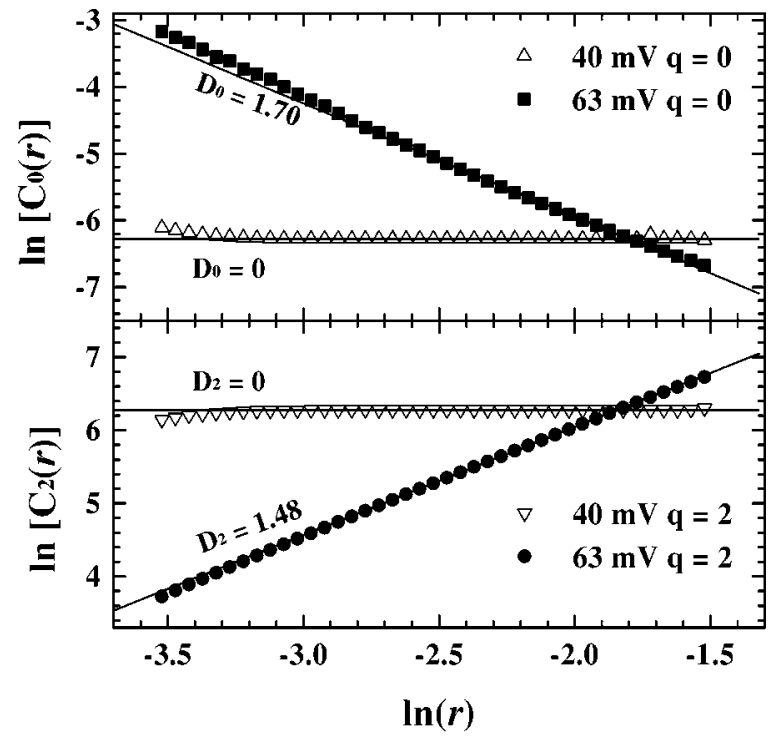

FIG. 1. $\ln \left[C_{q}(r)\right]$ vs $\ln (r)$ for $q=0$ and 2 at $V_{a c}=40$ and $63 \mathrm{mV}$, respectively. The solid lines are linear fits to the data points.

sion, $D_{1}$ as the information dimension, and $D_{2}$ as the correlation dimension. In fact, the multifractal dimension in this method is calculated through the generalized probability to find any two points of the attractor within a distance $r$, which is easier to compute in practice and gives smooth curves as compared to the grid method. ${ }^{16}$

As $q$ is varied, different regions of the attractor will determine $D_{q} . D_{\infty}$ corresponds to the region where the points are mostly concentrated, while $D_{-\infty}$ is determined by the region where the points have the least probability to be found. ${ }^{3}$ If $D_{q}$ is a constant for all $q$, then the trajectory will visit different parts of the attractor with the same probability, i.e., the point density is uniform in the Poincare map. This type of attractor is a so-called "trivial" attractor. When $D_{q}$ changes with $q$ reflecting a different point density among the attractor, it is denoted a "nontrivial" attractor. Therefore, the $D_{q}$ curves can be used to determine the structure of the attractor. In the following, we will compute $D_{q}$ curves from the experimentally obtained Poincaré maps shown in Fig. 3 of Ref. 13. We will then discuss the structure of the different attractors observed in the bifurcation route to chaos.

Figure 1 shows several $\ln \left[C_{q}(r)\right]$ vs $\ln (r)$ curves for 40 and $63 \mathrm{mV}$, which are calculated from the corresponding Poincaré maps. In this figure we only give examples for $q=0$ and 2. In these curves, a linear region is found for a certain range of $r$ values. For smaller and larger $r$, the curves depart from a linear dependence due to the finite number of points in the Poincare map and the finite size of the attractor. Although the limit $r \rightarrow 0$ is not very well fulfilled because in our experiment the number of the points in the Poincare map is limited to 1600 , the dimension of the attractors can still be estimated according to Eq. (1) by determining the slope of the linear region in Fig. 1. For $40 \mathrm{mV}$, the fractal dimensions $D_{0}$ and $D_{2}$ obtained in this way are approximately equal to zero, which is in agreement with a frequency-locked attractor. However, for the chaotic attractor at $63 \mathrm{mV}$, the dimensions $D_{0}=1.70$ and $D_{2}=1.48$ are fractal. They even vary with $q$.

Figure 2(a) shows the $D_{q}$ vs $q$ curves for the bifurcation 


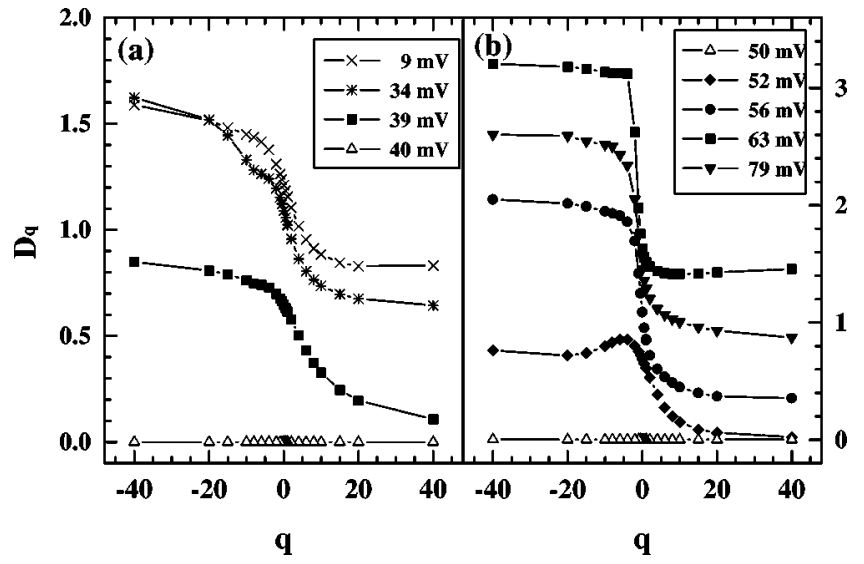

FIG. 2. Multifractal dimensions $D_{q}$ vs $q$ for the driving amplitudes $V_{a c}$ from 9 to $40 \mathrm{mV}$ (a) and from 50 to $79 \mathrm{mV}$ (b) obtained from the corresponding Poincaré maps.

sequence from quasiperiodicity $\rightarrow$ synchronized chaos $\rightarrow$ frequency locking (1 to $40 \mathrm{mV}$ ). For the frequency-locked state at $40 \mathrm{mV}$, the multifractal dimension $D_{q}$ is approximately equal to zero for all values of $q$ in good agreement with the corresponding attractor of three isolated points. The attractor for frequency locking is therefore not strange. For quasiperiodicity, the Poincare map should be a smooth loop. ${ }^{17}$ Consequently, it is expected that a quasiperiodic attractor is one dimensional, i.e., $D_{q}$ is equal to one for all values of $q$. However, the $D_{q}$ curves for the quasiperiodic attractors in our experiments (between $V_{a c}=1$ and $37 \mathrm{mV}$ ) do not exhibit a horizontal line as shown for 9 and $34 \mathrm{mV}$ in Fig. 2(a), although $D_{0}$ is close to one. $D_{-\infty}$ is about 1.6 and $D_{\infty}$ approaches a finite value below one. The "kneelike" structure of $D_{q}$ shown in Fig. 2(a) is typical for chaotic attractors with multifractal dimensions. We therefore conclude that the quasiperiodic attractors are nontrivial in our system. This can also be seen from the Poincare maps in Fig. 3 of Ref. 13, since there appears to be in some parts a doublelayer structure of the loop. Strange attractors are not always connected with chaos. The possibility of their emergence in nonchaotic systems has been predicted theoretically by Grebogi et al. ${ }^{18}$ and they have been experimentally observed in different physical systems, such as quasiperiodically forced systems ${ }^{19}$ and an undriven gas-discharge plasma, ${ }^{20}$ etc. Although there is no complete theory of such a behavior, usually the appearance of nonchaotic strange attractors is related to the intermittency induced by noise. ${ }^{21}$ A possible mechanism of their emergence at the boundaries of mode-locked tongues is discussed in Ref. 22.

For synchronized chaos at $39 \mathrm{mV}$, the $D_{q}$ curve also shows a "kneelike" structure. In contrast to the quasiperiodic regime, $D_{-\infty}$ is less than 1 , while $D_{\infty}$ approaches zero. This dependence indicates that the synchronized chaos observed at 38-39 mV exhibits the characteristics of both chaos and frequency locking. However, the attractor for synchronized chaos has a capacity dimension $D_{0}$ of about 0.65 , which is less than that of the quasiperiodic attractors.

When $V_{a c}$ increases beyond $50 \mathrm{mV}$, an explosive bifurcation sequence to chaos is observed as discussed in Sec. II. Figure 2(b) gives several multifractal dimensions for the attractors in this bifurcation regime. At $50 \mathrm{mV}$, the oscillations

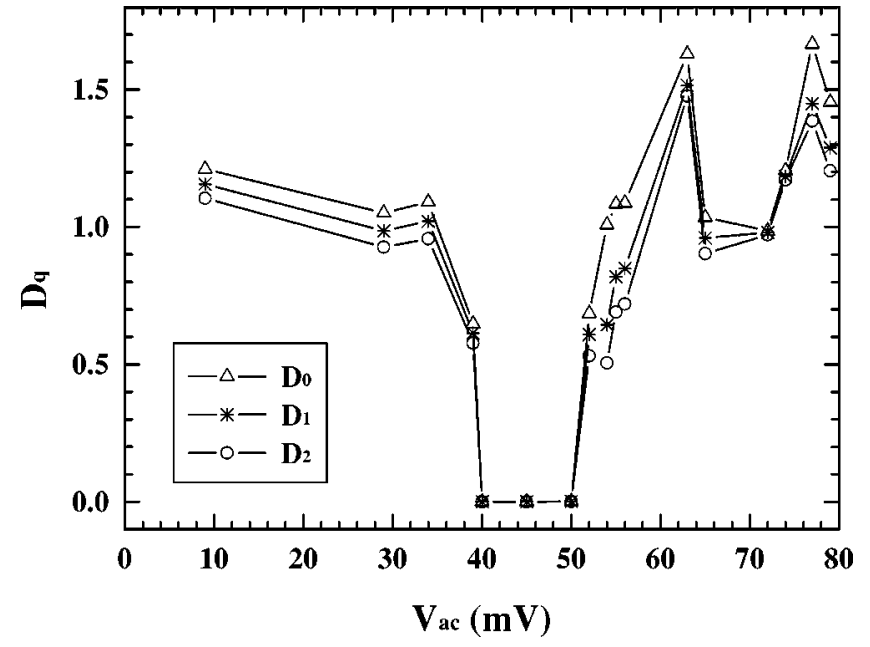

FIG. 3. Dimensions $D_{0}, D_{1}$, and $D_{2}$ as a function of the driving amplitude $V_{a c}$.

are periodic so that the dimension of the attractor is about zero. This is actually the case for all values of $V_{a c}$ between 40 and $50 \mathrm{mV}$. For $V_{a c}$ larger than $50 \mathrm{mV}$, the Poincare maps expand into more complicated structures. At the same time, the $D_{q}$ curves become nontrivial exhibiting the typical kneelike structure of strange attractors. With increasing $V_{a c}$ from 50 to $56 \mathrm{mV}$, the height of the kneelike structure increases indicating that the attractor becomes more strange. The system enters a chaotic regime with higher complexity. Additionally, the strangeness for the chaotic attractors in this regime is more pronounced than for synchronized chaos at $39 \mathrm{mV}$ and also for the quasiperiodic regime. This can be easily seen in Fig. 2(b), since the absolute values of $D_{q}$ as well as the height of the kneelike structure are larger than in Fig. 2(a). A number of chaotic attractors with different multifractal dimensions are observed between 56 and $80 \mathrm{mV}$. Figure 2(b) gives examples for 56, 63, and $79 \mathrm{mV}$.

Figure 3 shows the dependence of $D_{0}, D_{1}$, and $D_{2}$ on $V_{a c}$. It is very clear from this graph that the multifractal dimension of the attractor is changing during the bifurcation sequence to chaos. The dimensions $D_{0}, D_{1}$, and $D_{2}$ do not change very much within the quasiperiodic regime. When the frequency-locked state is reached at $40 \mathrm{mV}$, all three fractal dimensions vanish. Between 50 and $63 \mathrm{mV}$, all three fractal dimensions increase abruptly reaching slightly different maximum values of about 1.5 at $60 \mathrm{mV}$. The abrupt increase of the fractal dimensions demonstrates that the bifurcation sequence from frequency locking to chaos is explosive. Between 51 and $80 \mathrm{mV}$, a number of strange attractors is observed in the chaotic regime with varying fractal dimensions. Since for random noise the capacity dimension $D_{0}$ is equal to 2 (for the two-dimensional phase space considered here), we conclude that the observed dimension $D_{0}$ between 51 and $80 \mathrm{mV}$ with values of less than 2 clearly demonstrates the existence of deterministic low-dimensional chaos in our experiments.

\section{SUMMARY}

We have calculated the multifractal dimension of the attractors in an experimentally observed bifurcation sequence 
to chaos for a driven, weakly coupled semiconductor SL. These dimensions are obtained from Poincaré maps, which are constructed from real-time current traces and measure the strength of the multifractality of the corresponding attractor. The dependence of the fractal dimension on the driving voltage amplitude demonstrates quantitatively that the bifurcation sequence contains quasiperiodic, periodic, and chaotic attractors. Furthermore, the transition from frequency locking to chaos is explosive. In the quasiperiodic regime, the attractors may exhibit a certain degree of strangeness. Fi-

*Electronic address: htg@pdi-berlin.de

${ }^{1}$ P. Bergé, Y. Pomeau, and C. Vidal, Order Within Chaos (Wiley, New York, 1984).

${ }^{2}$ E. Ott, Chaos in Dynamical Systems (Cambridge University Press, New York, 1993).

${ }^{3}$ P. Grassberger, Phys. Lett. 97A, 227 (1983).

${ }^{4}$ H. G. E. Hentschel and I. Procaccia, Physica D 8, 435 (1983).

${ }^{5}$ L. L. Bonilla, in Nonlinear Dynamics and Pattern Formation in Semiconductors and Devices, edited by F.-J. Niedernostheide (Springer-Verlag, Berlin, 1995), Chap. 1.

${ }^{6}$ H. T. Grahn, in Hot Electrons in Semiconductors, Physics and Devices, edited by N. Balkan (Clarendon Press, Oxford, 1998), pp. 357-382.

${ }^{7}$ J. Kastrup, R. Hey, K. H. Ploog, H. T. Grahn, L. L. Bonilla, M. Kindelan, M. Moscoso, A. Wacker, and J. Galán, Phys. Rev. B 55, 2476 (1997).

${ }^{8}$ H. T. Grahn, R. J. Haug, W. Müller, and K. Ploog, Phys. Rev. Lett. 67, 1618 (1991).

${ }^{9}$ J. Kastrup, H. T. Grahn, K. Ploog, F. Prengel, A. Wacker, and E. Schöll, Appl. Phys. Lett. 65, 1808 (1994).

${ }^{10}$ H. T. Grahn, J. Kastrup, K. Ploog, L. L. Bonilla, J. Galán, M. Kindelan, and M. Moscoso, Jpn. J. Appl. Phys., Part 1 34, 4526 (1995). nally, the observed fractal dimensions unambiguously identify the existence of strange attractors and thereby confirm the deterministic nature of the observed chaotic oscillations.

\section{ACKNOWLEDGMENTS}

The authors would like to thank A. Fischer for sample growth. Partial support of the Deutsche Forschungsgemeinschaft within the framework of Sfb 296 is gratefully acknowledged.

${ }^{11}$ O. M. Bulashenko and L. L. Bonilla, Phys. Rev. B 52, 7849 (1995); O. M. Bulashenko, M. J. García, and L. L. Bonilla, ibid. 53, 10008 (1996).

${ }^{12}$ Y. Zhang, J. Kastrup, R. Klann, K. H. Ploog, and H. T. Grahn, Phys. Rev. Lett. 77, 3001 (1996); Y. Zhang, R. Klann, H. T. Grahn, and K. H. Ploog, Superlattices Microstruct. 21, 565 (1997).

${ }^{13}$ K. J. Luo, H. T. Grahn, K. H. Ploog, and L. L. Bonilla, Phys. Rev. Lett. 81, 1290 (1998).

${ }^{14}$ P. Grassberger and I. Procaccia, Physica D 9, 189 (1983).

${ }^{15}$ K. Pawelzik and H. G. Schuster, Phys. Rev. A 35, 481 (1987).

${ }^{16}$ P. Grassberger, Phys. Lett. A 148, 63 (1990).

${ }^{17}$ K. J. Luo, H. T. Grahn, S. W. Teitsworth, and K. H. Ploog, Phys. Rev. B 58, 12613 (1998).

${ }^{18}$ C. Grebogi, E. Ott, S. Pelikan, and J. A. Yorke, Physica D 13, 261 (1984).

${ }^{19}$ W. L. Ditto, M. L. Spano, H. T. Savage, S. N. Rauseo, J. F. Heagy, and E. Ott, Phys. Rev. Lett. 65, 533 (1990).

${ }^{20}$ W. X. Ding, H. Deutsch, A. Dinklage, and C. Wilke, Phys. Rev. E 55, 3769 (1997).

${ }^{21}$ J. W. Shuai and K. W. Wong, Phys. Rev. E 57, 5332 (1998).

${ }^{22}$ P. Glendinning, Phys. Lett. A 244, 545 (1998). 